\title{
Highly Robust and Imperceptible Luminance Based Hybrid Digital Video Watermarking Scheme for Ownership Protection
}

\author{
Himanshu Agarwal ${ }^{1}$, Rakesh Ahuja ${ }^{2}$, S.S.Bedi ${ }^{3}$ \\ ${ }^{1,2}$ Department of CS\&IT, Moradabad Institute of Technology, Moradabad (U.P), India \\ ${ }^{3}$ Department of CS \& IT, MJP Rohilkhand University, Bareilly (U.P), India \\ E-mail: himanshu.agg2000@gmail.com
}

\begin{abstract}
In this paper a hybrid digital video watermarking scheme based on discrete wavelet transform and singular value decomposition is proposed. Unlike the most existing watermarking schemes, the used watermark is a gray scale image instead of a binary watermark. The watermark is embedded in the original video frames by first converted it into $\mathrm{YCbCr}$ color space and than decomposing the luminance part (Y component) into four sub-bands using discrete wavelet transform and finally the singular values of LL sub-band are shaped perceptually by singular values of watermark image. The experimental result shows a tradeoff between imperceptibility and resiliency against intentional attacks such as rotation, cropping, histogram stretching, JPEG compression on individual frames, Indeo5 video compression and unintentional attacks like frame swapping, frame averaging, frame insertion and different types of noise addition. Superiority of the proposed scheme is carried out by comparison with existing schemes to reveal its efficiency for practical applications.
\end{abstract}

Index Terms - Video watermarking, Wavelet transform, Singular Value Decomposition, Ownership protection

\section{INTRODUCTION}

Due to the rapid development of the internet and the distribution of digital media through it, the digital data can be reproduced very easily which drives to urgent need to resolve ownership protection issues. Therefore, the field of digital watermarking grows extremely fast in these few years. Recently the video watermarking problem has generated a flurry of research activity in the area of digital watermarking of electronic content for ownership protection. A powerful watermarking scheme usually poses the following key properties [1]: (1) imperceptibility, (2) robustness, (3) capacity (4) unambiguity. But it is impossible to design a scheme which covers all features at once. Thus we make tradeoffs between aforementioned properties. The watermark used in the proposed video watermarking scheme is a gray scale logo instead of a binary logo due to its relevance in many practical situations and also the gray scale watermark has grater possibilities of survival under a number of popular attacks because gray-scale watermark carries a very high amount of watermark contents to preserve a certain degree of contextual relationship effect [2].

According to the processing methods video watermarking schemes mainly classified in two categories: Spatial domain and transform domain [3]. Spatial domain watermarking alters the pixel values of the original image directly. Such methods have very low computational complexity but they are vulnerable to attacks [3, 4]. In contrast the transform domain techniques, for example discrete cosine transformation (DCT), discrete wavelength transformation (DWT) and singular value decomposition (SVD) proved to be more robust and imperceptible due to disperse of watermark making it difficult for attacker to extract the embedded watermark [5].

Lama et al. [5] design the video watermarking algorithms using SVD transform and achieving fair level of imperceptibility but fail to achieve reasonable robustness against most of the attacks. Radu and Dragos [6] proposed video watermarking scheme in wavelet domain. They embed the watermark in wavelet coefficient of LH, HL and $\mathrm{HH}$ sub-bands of second wavelet decomposition and achieve some resilience against different attacks in the spatial and compressed domain. But the shortcoming of this method is that embedding watermark in multiple sub-bands of the wavelet domain is not well suited for video applications. Ming et al. [7] also design a video watermarking scheme based on MPEG-2 but it is limited to low bit rate MPEG2 compression and synchronization attacks. Our paper shows a combination of discrete wavelet transforms and the singular value decomposition schemes to provide better results as compared to use them individually, by using the following advantages inherited with SVD and DWT [5]: 1) If the watermark is embedded in the low frequencies bands of wavelet transform than it provide some resilience against compression and when embed in the high frequencies bands it provide robustness against noise addition and several type of filters. Wavelets are easily adoption for human visual systems. 3) SVD is widely use in watermarking applications for improving the robustness. 
Till now the techniques used for video watermarking are lagging behind, because of poor anti-attack technology and characteristics of the digital products that they are easy to copy and transfer. Facing the popularity of communication networks, digital video ownership protection and authentication is the critical issues which need to be solved. The proposed video watermarking scheme is an effective technique to solve these problems by retaining imperceptibility and robustness against various attacks.

The rest of the paper is organized as follows. In section two, we briefly introduce the concept of SVD and DWT transformation techniques for understanding our scheme. In section three, we propose our video watermarking method, where we describe the watermark embedding and extraction process. In section four, we presents the experimental results and in section five, same are compare with existing work done by previous researchers. The conclusions of our study are stated in section six.

\section{PRELIMINARIES}

Since the details of the SVD and DWT are found in Refs. [2] and [8], this paper gives only a brief overview as below.

\section{A. Singular Value Decomposition (SVD)}

SVD is extensively used in a variety of image processing applications because it reveals several essential properties: 1) SVD of an image does not change significantly when a small perturbation is added to the image. Because of this property SVD has been used in watermarking methods to improving the robustness. 2) The SVD image matrix containing many small values compared with the first singular value so if we neglect these smaller values in reconstruction of an image, it does not degrade the quality of the image. Due to this reason it is widely used in compression of images. 3) Singular value of an image specifies the brightness of an image layer and the singular vector represents the geometry of the image.

The SVD technique decomposes a matrix A of size $M$ $\mathrm{x} \mathrm{N}$ in three matrices: $\mathrm{U}, \mathrm{S}$ and $\mathrm{V}$ according to the following equation,

$$
\begin{aligned}
\mathrm{A} & =\mathrm{U} \times \mathrm{S} \times \mathrm{V}^{\mathrm{T}} \\
& =\left[\begin{array}{cccc}
u_{1,1} & . . & . . & u_{1, M} \\
u_{2,1} & . . & . . & u_{2, M} \\
: & : & : & : \\
: & : & : & : \\
u_{M, 1} & . . & . . & u_{M, M}
\end{array}\right] \times\left[\begin{array}{cccc}
\sigma_{1,1} & 0 & . . & 0 \\
0 & \sigma_{2,2} & . . & 0 \\
: & : & : & : \\
: & : & : & : \\
0 & 0 & . . & \sigma_{N, N}
\end{array}\right]
\end{aligned}
$$

$$
\mathrm{x}\left[\begin{array}{cccc}
v_{1,1} & . . & . . & v_{1, N} \\
v_{2,1} & . . & . . & v_{2, N} \\
: & : & : & : \\
: & : & : & : \\
v_{N, 1} & . . & . . & v_{N, N}
\end{array}\right]^{T}
$$

where $\mathrm{U}$ and $\mathrm{V}$ given in Eq.(1) are the orthogonal matrices of size $\mathrm{M} \times \mathrm{M}$ and $\mathrm{N} \times \mathrm{N}$ respectively, with the properties of $U_{T} U=I$ and $V_{T} V=I$, here $I$ is the identity matrix. $\mathrm{S}$ is the diagonal matrix of $\mathrm{Mx} \mathrm{N}$ containing the eigen values $\left(\sigma_{i, i}\right)$ of matrix $\mathrm{A}$ arranged in a descending order.

\section{B. Discrete Wavelet Transform (DWT)}

DWT is an important tool for multiresolution decomposition of images or video frames and are widely used in image and video processing due to its following inherited benefits over the sinusoids based transform:

1) It has an ability to analyze the signal in both time and frequency domain simultaneously.

2) Wavelet exhibit constant shape because they are generated from only one function

In wavelet investigation, an orthonormal set of functions, as well as a nonorthogonal, but linearly independent basis are employed [2]. A 2D wavelet transform decompose an image into four components:

1) Approximation Coefficients

2) Horizontal Coefficients

3) Vertical Coefficients

4) Diagonal Coefficients

Approximation coefficients are also called as base image which represents the low frequency part. The horizontal, vertical and diagonal coefficients are called detail coefficients and represent the high frequency part along horizontal, vertical and diagonal of the image.

Let I be an image (or frame of an video), after decomposed by wavelet transformation, the consequent of the first level decomposition is

$$
\mathrm{I}=\mathrm{Iw}_{\mathrm{LL} 1}+\mathrm{Iw}_{\mathrm{LH} 1}+\mathrm{Iw}_{\mathrm{HL} 1}+\mathrm{Iw}_{\mathrm{HH} 1}
$$

where $\mathrm{Iw}_{\mathrm{LL} 1}$ represent the base image (approximation) and $\mathrm{Iw}_{\mathrm{LH} 1}, \mathrm{Iw}_{\mathrm{HL} 1}, \mathrm{Iw}_{\mathrm{HH} 1}$ are the first level vertical, horizontal and diagonal details. The approximation and detail images obtained from decomposition are usually structured as shown in Fig. 1.

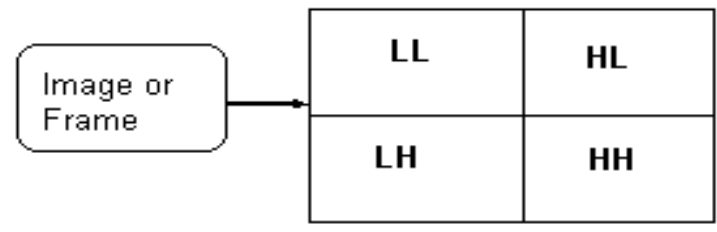

Fig. 1 Wavelet Decomposition 
We can get the nth level decomposition by recursively applying 2D wavelet on individual coefficients of the derived components to get more fine details of the image (frame).

\section{Proposed Watermarking Scheme}

In the propose method, we take the size of the watermark is equal to the approximation part of the first level decomposition of the image, derived by applying discrete wavelet transform on the original image.

\section{A. Embedding Procedure}

Step 1. Convert the RGB video frame into $\mathrm{YCbCr}$ color space by using the following equations,

$\mathrm{Y}=0.299 \times \mathrm{R}+0.587 \times \mathrm{G}+0.114 \times \mathrm{B}$

$\mathrm{Cb}=0.596 \times \mathrm{R}-0.275 \times \mathrm{G}-0.321 \times \mathrm{B}$

$\mathrm{Cr}=0.212 \times \mathrm{R}-0.523 \times \mathrm{G}-0.311 \times \mathrm{B}$

Step 2. Decompose the luminance part of the frame by apply DWT, resulting in approximation part LL1 and detail parts LH1, HL1 and HH1 respectively.

Step 3. Decompose the approximation part (LL1 subband) of DWT by apply singular value decomposition, resulting $\mathrm{U}_{\mathrm{F}}, \mathrm{S}_{\mathrm{F}}$ and $\mathrm{V}_{\mathrm{F}}$ matrices, where $S_{F}$ represents the singular values of approximation part in descending order.

$\mathrm{SVD}(\mathrm{LL} 1)=\mathrm{UF} \times \mathrm{SF} \times \mathrm{VF}$

Step 4. Apply singular value decomposition on the watermark $\mathrm{W}$, by which watermark is decomposed into three matrices $\mathrm{U}_{\mathrm{W}}, \mathrm{S}_{\mathrm{W}}$ and $\mathrm{V}_{\mathrm{W}}$, where $S_{W}$ contains the singular values of the watermark in descending order.

$\operatorname{SVD}(\mathrm{W})=\mathrm{UW} \times \mathrm{SW} \times \mathrm{VW}$

Step 5. Modify the singular values of the approximation part by singular values of the watermark as,

$\mathrm{SF}^{*}=\mathrm{SF}+\alpha \mathrm{SW}$

where $\alpha$ is the scale coefficient, to balance the tradeoff between the robustness and imperceptibility. Its value is taken as 0.01 in this paper.

Step 6. For getting the modified wavelet approximation coefficient, inverse SVD is applied on the modified singular values $\mathrm{S}_{\mathrm{F}} *$ and $\mathrm{U}_{\mathrm{W}}, \mathrm{V}_{\mathrm{W}}$ values obtained from Eq. (4).

Step 7. Apply inverse DWT on the modified approximation part and all detail parts to get the modified luminance part $\mathrm{Y}^{\prime}$ of the frame.

Step 8. Reconstruct the video frame (watermarked video frame) from the modified luminance part $\mathrm{Y}^{\prime}$ and chrominance part $\mathrm{Cb}$ and $\mathrm{Cr}$ of the original frame by convert the $\mathrm{YCbCr}$ into RGB color space using the following equations,

$$
\begin{aligned}
& \mathrm{R}^{\prime}=\mathrm{Y}^{\prime}+0.956 \times \mathrm{Cb}+0.620 \times \mathrm{Cr} \\
& \mathrm{G}^{\prime}=\mathrm{Y}^{\prime}-0.272 \times \mathrm{Cb}-0.647 \times \mathrm{Cr} \\
& \mathrm{B}^{\prime}=\mathrm{Y}^{\prime}-1.108 \times \mathrm{Cb}+1.705 \times \mathrm{Cr}
\end{aligned}
$$

\section{B. Extraction Procedure}

Step 1. Convert the RGB video frame into $\mathrm{YCbCr}$ color space.

Step 2. Decompose the luminance part of the frame by apply DWT, resulting in approximation part LL1 and detail parts LH1, HL1 and HH1.

Step 3. Decompose the approximation part (LL1 subband) of DWT by apply singular value decomposition, resulting $\mathrm{U}_{\mathrm{F}}, \mathrm{S}_{\mathrm{F}} *$ and $\mathrm{V}_{\mathrm{F}}$ matrices, ad getting the singular values of approximation part $\mathrm{S}_{\mathrm{F}}{ }^{*}$.

Step 4. Extract singular values of the watermark from approximation part,

$\mathrm{Sw}^{*}=\left(\mathrm{SF}^{*}-\mathrm{SF}\right) / \alpha$

Step 5. Extract the watermark image $\mathrm{W}^{\prime}$ by using the inverse SVD as

$\mathrm{W}^{\prime}=\mathrm{UW} \times \mathrm{Sw}^{*} \mathrm{x} \mathrm{VTW}$

\section{EXPIRENMENTAL SETUP AND RESULTS}

The imperceptibility and robustness of the proposed video watermarking scheme is demonstrated using MATLAB by considering standard color video sequence Rhino in $240 \times 320$ pixels with the frame rate of $15 \mathrm{fps}$ and a length of 114 frames. The two standard gray-scale images logo.tif and cameraman.tif are of same size $120 \mathrm{x}$ 160 pixels (by resizing it so that its size is equal to the size of LL band of the video frame) are selected as watermark. Transparency (imperceptibility) of the watermark is estimated by PSNR (peak signal to noise ratio). If the PSNR is higher than we get the less distorted frame. PSNR is measured in decibels $(\mathrm{dB})$ as:

$$
P S N R=10 \log _{10} \frac{f_{\mathrm{P} e a k}^{2}}{M S E}
$$

where MSE is mean square error between the watermarked frame, $f_{\mathrm{w}}$ and the original one, $f_{\mathrm{o}}$. The MSE is given as

$$
\operatorname{MSE}=\frac{\sum_{\mathrm{i}=1}^{\mathrm{N}_{1}} \sum_{\mathrm{j}=1}^{\mathrm{N}_{2}}\left[\mathrm{f}_{\mathrm{o}}(\mathrm{i}, \mathrm{j})-\mathrm{f}_{\mathrm{w}}(\mathrm{i}, \mathrm{j})\right]^{2}}{\mathrm{~N}_{1} \times \mathrm{N}_{2}}
$$

Fig. 2 shows the tenth frame in the original video with the corresponding watermarked and attacked frames. Through figure we can conclude that frames can not be distinguished by the Human Visual System (HSV).

Robustness which is the ability of a persistent association method to withstand signal modifications under different attacks is computed by the correlation coefficient $(C C)$ [10]. $C C$ between embedded gray-scale watermark image $(\mathrm{W})$ and extracted watermark image $\left(\mathrm{W}^{\prime}\right)$ is given as,

$$
C C=\frac{\sum_{m} \sum_{n}\left(W_{m n}-\bar{W}\right)\left(W_{m n}^{\prime}-\bar{W}^{\prime}\right)}{\sqrt{\left(\sum_{m} \sum_{n}\left(W_{m n}-\bar{W}\right)^{2}\right)\left(\sum_{m} \sum_{n}\left(W_{m n}^{\prime}-\bar{W}^{\prime}\right)^{2}\right)}}
$$


where $\mathrm{W}$ and $\mathrm{W}^{\prime}$ are the image matrices of the same size and $\bar{W}$ and $\bar{W}^{\prime}$ are the mean value of embedded watermark image and extracted watermark image respectively. The original and extracted watermarks are shown in Fig. 3.

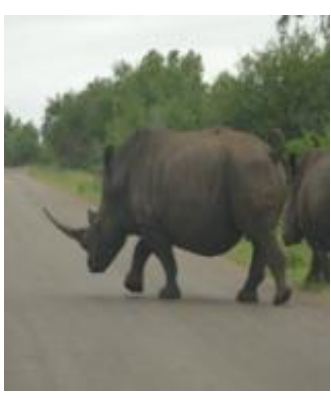

Fig. 2 (a) Original Frame

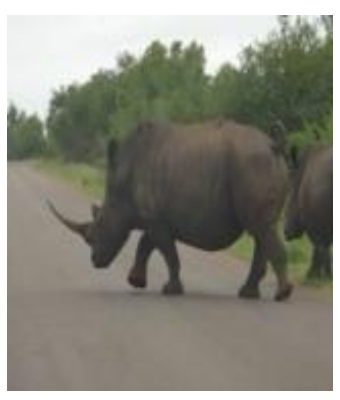

Fig. 2 (b)

Watermarked Frame

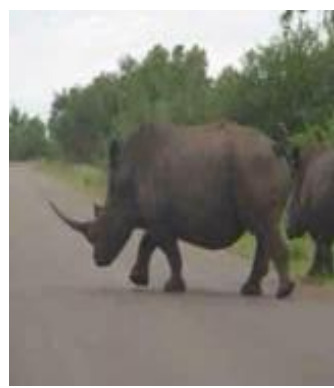

Fig. 2 (c). JPEG Compressed Frame $(\mathrm{Q}=25)$

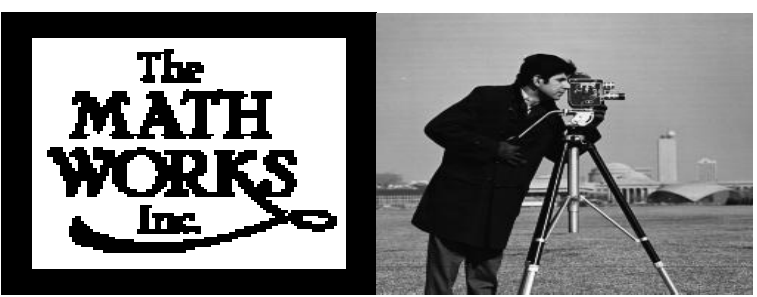

Fig. 3 (a) Original Watermarks

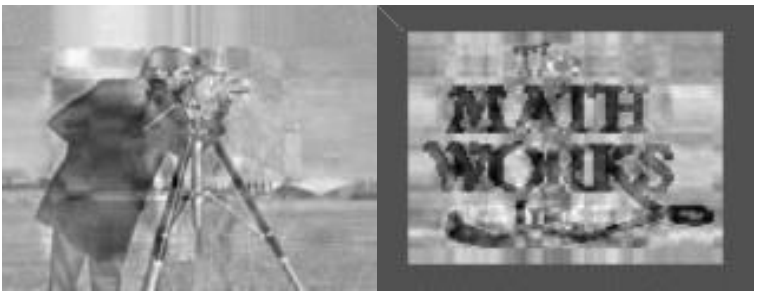

Fig. 3 (b) Extracted Watermarks. JPEG Compression $(\mathrm{Q}=25)$

Results of the Test obtained are summarized in Table:
TABLE I PSNR VALUE OF COVER VIDEO AND CORRELATION COEFFICIENT OF EXTRACTED WATERMARK (LOGO.TIFF) UNDER VARIOUS ATTACKS

\begin{tabular}{|c|c|c|c|}
\hline Attack Type & $\begin{array}{l}\text { PSNR } \\
\text { Value } \\
\end{array}$ & $\mathbf{C C}$ & Extracted Watermarl \\
\hline $\begin{array}{c}\text { Cropping } \\
\text { (40 Columns) }\end{array}$ & 19.2870 & 0.9218 & \\
\hline $\begin{array}{c}\text { Rotation } \\
\left(0.5^{\circ} \text { Degree }\right)\end{array}$ & 31.5955 & 0.8913 & \\
\hline $\begin{array}{c}\text { Salt and } \\
\text { Peeper } \\
\text { Density } 0.01\end{array}$ & 29.8433 & 0.6948 & \\
\hline $\begin{array}{l}\text { Speckle } \\
\text { Noise } \\
\text { Variance } \\
0.001\end{array}$ & 33.5889 & 0.8699 & \\
\hline $\begin{array}{l}\text { Poisson } \\
\text { Noise }\end{array}$ & 35.1739 & 0.8593 & \\
\hline $\begin{array}{c}\text { JPEG } \\
\text { Compression } \\
\text { Quality } \\
\text { Factor } Q=25\end{array}$ & 33.9343 & 0.8691 & \\
\hline $\begin{array}{c}\text { Indeo5 } \\
\text { Compression } \\
\text { Quality } \\
\text { Factor } Q=25\end{array}$ & 33.5694 & 0.8691 & \\
\hline $\begin{array}{c}\text { Frame } \\
\text { Swapping } \\
\text { (Swap Six } \\
\text { Frames) }\end{array}$ & 32.7610 & 0.8530 & \\
\hline $\begin{array}{c}\text { Frame } \\
\text { Averaging } \\
\text { (Average } \\
\text { Two Frames) }\end{array}$ & 32.9146 & 0.8579 & \\
\hline $\begin{array}{c}\text { Frame } \\
\text { Insertion } \\
\text { (Ten Frames } \\
\text { Inserted) }\end{array}$ & 24.1094 & 0.9173 & 200 \\
\hline $\begin{array}{c}\text { Intensity } \\
\text { Adjustment } \\
\text { Between } \\
{[.2 .3 .1] \text { to }} \\
{\left[\begin{array}{lll}6 & .7 & 1\end{array}\right]}\end{array}$ & 21.1467 & 0.9645 & \\
\hline
\end{tabular}


TABLE II. PSNR VALUE OF COVER VIDEO AND CORRELATION COEFFICIENT OF EXTRACTED WATERMARK (CAMERAMAN.TIFF) UNDER VARIOUS ATTACKS

\begin{tabular}{|c|c|c|c|}
\hline Attack Type & $\begin{array}{l}\text { PSNR } \\
\text { Value }\end{array}$ & $\mathrm{CC}$ & Extracted Watermark \\
\hline $\begin{array}{l}\text { Cropping } \\
\quad(40 \\
\text { Columns })\end{array}$ & 19.2875 & 0.9057 & \\
\hline $\begin{array}{c}\text { Rotation } \\
\left(0.5^{\circ} \text { Degree }\right)\end{array}$ & 31.6002 & 0.8500 & \\
\hline $\begin{array}{c}\text { Salt and } \\
\text { Peeper } \\
\text { Density } 0.01\end{array}$ & 29.8398 & 0.6061 & \\
\hline $\begin{array}{c}\text { Speckle } \\
\text { Noise } \\
\text { Variance } \\
0.001\end{array}$ & 33.6066 & 0.8394 & \\
\hline $\begin{array}{l}\text { Poisson } \\
\text { Noise }\end{array}$ & 35.1980 & 0.8655 & \\
\hline $\begin{array}{c}\text { JPEG } \\
\text { Compression } \\
\text { Quality } \\
\text { Factor } Q=25\end{array}$ & 33.9736 & 0.8434 & \\
\hline $\begin{array}{c}\text { Indeo5 } \\
\text { Compression } \\
\text { Quality } \\
\text { Factor } \mathrm{Q}=25\end{array}$ & 33.8426 & 0.8426 & \\
\hline $\begin{array}{l}\text { Frame } \\
\text { Swapping } \\
\text { (Swap Six } \\
\text { Frames) }\end{array}$ & 32.8024 & 0.8316 & \\
\hline $\begin{array}{c}\text { Frame } \\
\text { Averaging } \\
\text { (Average } \\
\text { Two Frames) }\end{array}$ & 32.9579 & 0.8368 & \\
\hline $\begin{array}{c}\text { Frame } \\
\text { Insertion } \\
\text { (Ten Frames } \\
\text { Inserted) }\end{array}$ & 24.1123 & 0.9110 & \\
\hline 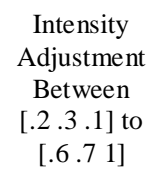 & 21.1473 & 0.9660 & \\
\hline
\end{tabular}

\section{Comparison With The Previous Work}

Results obtained from the Table. I and Table. II are compared with the existing work which shows in Table. III. Superiority of the proposed scheme is carried out by comparison made with existing schemes which reveal the efficiency of proposed scheme for practical applications.

TABLE III. COMPARISON BETWEEN CORRELATION COEFFICIENTS OF THE PROPOSED SCHEME AND THE EXISTING METHODS

\begin{tabular}{|c|c|c|c|c|c|}
\hline Attack Type & {$[11]$} & {$[12]$} & {$[13]$} & {$[14]$} & $\begin{array}{c}\text { Proposed } \\
\text { Scheme }\end{array}$ \\
\hline $\begin{array}{c}\text { JPEG } \\
\text { Compression }\end{array}$ & 0.812 & 0.510 & 0.65 & - & 0.8691 \\
\hline $\begin{array}{c}\text { Frame } \\
\text { Averaging }\end{array}$ & 0.661 & 0.482 & - & - & 0.8579 \\
\hline $\begin{array}{c}\text { Contrast } \\
\text { Adjustment }\end{array}$ & - & - & - & 0.5192 & 0.9645 \\
\hline
\end{tabular}

\section{CONCLUSIONS}

In this paper we proposed a robust video watermarking scheme for ownership protection based on wavelet transform and singular valued decomposition. Eleven different types of attacks were applied to the watermarked video in the proposed system. Simulation results shows that the proposed scheme achieve good resilience against most of the geometric and video specific attacks. A comparison with different algorithms reveals the superiority of the proposed scheme.

According to the experimental results summarized in the Table. 1 and Table. 2 above, the proposed watermarking scheme can achieved the following features:

1) In the proposed scheme, we embed the watermark on the SVD coefficients achieved by taking LL band through DWT of video frame, so the scheme has certain robustness and it preserve the transparency of the watermark in the video sequence.

2) The proposed scheme also shows considerable amount of resistance against the various types of noise even if the watermark is shaped in the low frequencies of the video frame through DWT.

3) The capacity of embedding is good enough as the size of the embedded watermark is greater than the size of watermarks use by most of the previous researchers $[2,9]$.

\section{ACKNOWLEDGMENT}

The authors would like to thank Mr. Farooq Husian Associate professor, Moradabad Institute of Technology, Moradabad (U.P) India for the careful reading of the paper and for constructive, focused comments and suggestions. 


\section{REFERENCES}

[1] Jamal Ali Hussein, "Luminance-based Embedding Approach for Color Image Watermarking", I.J. Image, Graphics and Signal Processing, 2012, 3, 4955

[2] Gaurav Bhatnagar, Q. M. Jonathan Wu, Balasubramanian Raman, "Robust gray-scale logo watermarking in wavelet domain", Computers and Electrical Engineering 3 (2012), doi 10.1016/j.compeleceng.2012.02.002.

[3] N. Nikolaidis, I. Pitas, "Robust image watermarking in the spatial domian", Signal Processing 66 (3) (1998) 385-403.

[4] J. C. Liu, S. Y. Chen, "Fast two layer image watermarking without referring to the original image and watermark", Image and Vision Computing 19 (14) (2001) 1083-1097.

[5] Lama Rajab, Tahani Al-Khatib, Ali Al-Haj "Video Watermarking Algorithms Using the SVD Transform", European Journal of Scientific Research, ISSN 1450-216X Vol.30 No.3 (2009), pp.389-401.

[6] Radu O. Preda, Dragos N. Vizireanu, "A robust digital watermarking scheme for video copyright protection in wavelet domain", Measurement 43 (2010) 1720-1726.

[7] Ming-Shi Wang, Wei-Che Chen, "A hybrid DWTSVD copyright protection scheme based on k-means clustering and visual cryptography",Computer Standards \& Interfaces 31 (2009) 757-762.

[8] Guoliang Zeng, "Face Recognition with Singular Value Decomposition”, CISSE Proceeding,2006.

[9] Saeed Rastegara, Fateme Namazi, Khashayar Yaghmaie, Amir Aliabadian, "Hybrid watermarking algorithm based on Singular Value Decomposition and Radon transform", Int. J. Electron. Commun. (AEÜ) 65 (2011) 658-663.

[10] J. Zhang, A. Ho, G. Qiu, P. Marziliano, Robust video watermarking of H./ AVC, IEEE Transactions on Circuits and System-II : Express Briefs 54(February) (2007) 205-209.

[11]X. Niu and S. Sun, "A New Wavelet-Based Digital Watermarking for Video," in Proceedings of the 9th IEEE Digital Signal Processing Workshop, 2000, pp. 241-245.

[12]C. Hsu, "DCT-Based Watermarking for Video," IEEE Trans. Consumer Electronics, vol. 44, no. 1, pp. 206-216, Jan. 1998.

[13]M. R. Saghavi, A. M. Rajukar, R. Mathur, K. S. Kotecha, "A Robust scheme for digital video watermarking based on scrambling of watermark," I. J. Computer Applications Vol. 35 (2) Dec. 2011.

[14] S. Sinha, P. Bardhan, Swarnali. P, A. Jagatramka, D. K. kole, A. Chakarborty, "Digital Video watermarking using DWT and Principal component analysis," I. J. Wisdom based computing Vol.1 (2) Aug-2011.

Himanshu Agarwal, male, obtained his B.Tech degree in Computer Engineering \& Information Technology, from Uttar Pradesh Technical University, Lucknow, India. He received the M.Tech degree in Computer Science \& Engineering from Teerthankar Mahaveer University, Uttar Pradesh, India. He is currently an Assistant Professor at Moradabad Institute of Technology, Moradabad, Uttar Pradesh, India. His research interests include Digital Watermarking, High speed networks and Computer Graphics. His teaching interests include digital image processing, Computer Graphics, and Networking Technology.

Rakesh Ahuja, male, received his B.Tech degree from BIET Jhansi, (U.P) India, M.Tech Degree from Uttar Pradesh Technical University, India. He is currently an Associate Professor at Moradabad Institute of Technology, Moradabad, (U.P) India. He is having 17 years of experience in the area of industrial, academic and administration. He is currently pursuing the Ph.D. degree from IFTM University, (U.P) India majoring in development schemes of image processing. His research interests are Image and Video Watermarking.

Dr. S. S. Bedi, male, received B.E degree from Guru Nanak Dev Engineering Collge, Bidar, India, M.Tech Degree from Thapar University Patiala, Punjab, India and Ph.D Degree from IIIT, Gwalior, (M.P) India. He is having sixteen years of experience in the area of academics, research and administration at MJPR University, Bareilly (U.P). He is member of various professional societies such as International Association of Engineers-China, Computer Society of India and IEEEUSA. His research interests are Information Security and digital watermarking. He was honored by Rashtriya Shikshak Ratan Award, AIBDA, New Delhi and also honored for best paper at World Congress on Engineering, London. U.K. 